\title{
NONLINEAR BOUNDARY VALUE PROBLEMS FOR ELLIPTIC SYSTEMS
}

\author{
by A. CAÑADA \\ (Received 30th October 1985)
}

\section{Introduction}

The purpose of this paper is to discuss non-linear boundary value problems for elliptic systems of the type

$$
\begin{array}{rlrl}
A_{k} u_{k}-\lambda_{1}^{k} u_{k} & =g_{k}\left(x, u_{1}, \ldots, u_{m}\right), & & x \in G, \quad 1 \leqq k \leqq m \\
B_{k} u_{k} & =0, & x \in \partial G, 1 \leqq k \leqq m
\end{array}
$$

where $A_{k}$ is a second order uniformly elliptic operator and $\lambda_{1}^{k} \in R$ is such that the problem

$$
\begin{array}{ll}
A_{k} u_{k}=\lambda_{1}^{k} u_{k}, & x \in G \\
B_{k} u_{k}=0, & x \in \partial G
\end{array}
$$

has a one-dimensional space of solutions that is generated by a non-negative function. The boundary $\partial G$ is supposed to be smooth and the functions $g_{k}, 1 \leqq k \leqq m$, are defined on $\bar{G} \times R^{m}$ and are continuously differentiable (usually, $B_{k}$ represents Dirichlet or Neumann conditions and $\lambda_{1}^{k}$ is the first eigenvalue associated with $A_{k}$ and such boundary conditions).

We shall consider classical solutions of (1.1) and thus no previous assumptions concerning the growth of non-linearities $g_{k}$ are necessary. Moreover, this allows us to obtain some conditions of asymptotic nature which are expressed in terms of non-strict inequalities and extend to the vector case those given by Kazdan and Warner [10] and Landesman and Lazer [11]. At the same time, we generalize the main results in $[7,12]$.

In Section 2 we obtain a general existence theorem for equations in some normed spaces which is related to a previous theorem of Ortega and the author [5]. In Section 3 we apply the results of Section 2 to the problem (1.1) and we give some examples which show the obtained generalizations.

The main tool we use is the Leray-Schauder degree.

\section{Abstract results}

Let $X$ and $Z$ be normed spaces and $L: \operatorname{dom} L \subset X \rightarrow Z$ a linear Fredholm mapping of index zero. We assume that the spaces $X$ and $Z$ are included into some space of functions $L^{\infty}\left(G, R^{m}\right)$, where $G$ is a bounded domain in an euclidean space $R^{n}$. 
Let us consider the equation

$$
L u=N u
$$

where $N: X \rightarrow Z$ is a given mapping.

It can be shown that $u$ is a solution of this equation if and only if

$$
u=P u+K(I-Q) N u+J Q N u
$$

where $P: X \rightarrow X$ and $Q: Z \rightarrow Z$ are projectors such that $\operatorname{Im} P=\operatorname{ker} L, \operatorname{Im} L=\operatorname{ker} Q, K$ is the generalized inverse of $L$ and $J$ is an isomorphism from $\operatorname{Im} Q$ into $\operatorname{ker} L$. Suppose that the mappings $Q N: X \rightarrow Z$ and $K(I-Q) N: X \rightarrow X$ are compact on bounded subsets of $X$ (i.e., continuous and $Q N(B), K(I-Q) N(B)$ are relatively compact for each bounded $B \subset X)$ and that $K$ is continuous.

As a standing hypothesis on the $\operatorname{ker} L$ we have

$$
\operatorname{ker} L=\left\{\left(a_{1} \phi_{1}, \ldots, a_{m} \phi_{m}\right):\left(a_{1}, \ldots, a_{m}\right) \in R^{m}\right\}
$$

where the functions $\phi_{k}: \bar{G} \rightarrow R$ are continuous, positive on $G$ and

$$
\left|u_{k}(x)\right| \leqq c \phi_{k}(x)\|u\|_{X} \quad \forall x \in \bar{G}
$$

for all $u=\left(u_{1}, \ldots, u_{m}\right) \in X$ and each $k, 1 \leqq k \leqq m$ ( $c$ is a positive constant independent of $u$ and $k$ ). Also, we suppose that the functions $\phi_{k}$ are normalized in the sense that $\int_{G} \phi_{k}^{2}(x) d x=1$.

The main result is the following theorem.

Theorem 2.1. Suppose $(\mathrm{H})$ holds and that:

(i) There exists a mapping $\xi: X \rightarrow Z^{*}$ and constants $\alpha \geqq 0, \beta \geqq 0$, such that

$$
\|N u\|_{z} \leqq\langle N u, \xi u\rangle+\alpha\|u\|_{x}+\beta
$$

where $Z^{*}$ is the normed dual space of $Z$ and $\langle N u, \xi u\rangle=(\xi u)(N u)$ for every $u \in X$.

(ii) There exists a bounded ( $\Phi$ takes bounded sets into bounded sets) and continuous mapping $\Phi: X \rightarrow \operatorname{Im} Q$ such that

(ii.1) Every possible solution $u \in X$ of the equation

$$
L u=\lambda N u+(1-\lambda) \Phi u, \quad \lambda \in] 0,1[
$$

satisfies the relation $\langle N u, \xi u\rangle \leqq 0$.

(ii.2) There exists $r>0$ such that for all $u \in X, u=\left(u_{1}, \ldots, u_{m}\right)$ with $\left|u_{k}(x)\right| \geqq r \phi_{k}(x)$, $\forall x \in G$ for some $k, 1 \leqq k \leqq m$, one has for some $j, 1 \leqq j \leqq m$, that $(Q N)_{j}(u)(\Phi)_{j}(u) \geqq 0$ and that the second factor is not zero. 
(ii.3) $d_{B}\left(\left.J \Phi\right|_{\text {ker } L}, B_{X}(s) \cap \operatorname{ker} L, 0\right) \neq 0$ for every $s \geqq r$, where $d_{B}$ means the Brouwer degree and $B_{X}(s)$ is the open ball of centre zero and radius $s$ in $X$.

Then, if $\alpha$ is sufficiently small, equation (2.1) has at least one solution.

Proof. We know (from the Leray-Schauder degree theory) that equation (2.1) will have a solution if we prove that there exists an open bounded subset $\Omega$ of $X$ of the form $\Omega=B_{X}\left(r_{1}\right), r_{1} \geqq r$, such that

$$
u-T(u, \lambda) \equiv u-P u-\lambda K(I-Q) N u-\lambda J Q N u-(1-\lambda) J \Phi u \neq 0
$$

for each $\lambda \in[0,1[$ and each $u \in \partial \Omega$. In fact, if this is the case and $u-T(u, 1)=0$ for some $u \in \partial \Omega$, we have, from the equivalence between (2.1) and (2.2) that (2.1) has a solution. If $u-T(u, 1) \neq 0 \forall u \in \partial \Omega$, we deduce from the homotopy property of the Leray-Schauder degree that

$$
d_{\mathrm{L}-\mathrm{S}}(I-T(\cdot, 1), \Omega, 0)=d_{\mathrm{L}-\mathrm{S}}(I-T(\cdot, 0), \Omega, 0)=d_{\mathrm{L}-\mathrm{S}}(I-P-J \Phi, \Omega, 0) .
$$

But as $\operatorname{Im}(P+J \Phi) \subset \operatorname{ker} L$ is finite dimensional,

$$
\begin{aligned}
d_{\mathrm{L}-\mathrm{S}}(I-P-J \Phi, \Omega, 0) & =d_{B}\left(I-P-\left.J \Phi\right|_{\mathrm{ker} L}, \Omega \cap \operatorname{ker} L, 0\right) \\
& =d_{B}\left(-\left.J \Phi\right|_{\mathrm{ker} L}, \Omega \cap \operatorname{ker} L, 0\right)
\end{aligned}
$$

and this degree is different from zero because of (ii.3).

Also, we must remark that the previous assumptions do not depend upon the choice of $P$ (see [13]) and, for convenience, we take $P: X \rightarrow X$ defined by $(P u)_{k}(x)=$ $\phi_{k}(x) \int_{G} u_{k}(x) \phi_{k}(x) d x, 1 \leqq k \leqq m$.

To prove (2.4), let $u \in X$ and $\lambda \in] 0,1[$ such that

$$
u-P u=\lambda K(I-Q) N u+\lambda J Q N u+(1-\lambda) J \Phi u
$$

Then applying $P$ to both parts of $(2.5)$ we obtain

$$
\begin{gathered}
u-P u=\lambda K(I-Q) N u \\
\lambda J Q N u+(1-\lambda) J \Phi u=0 .
\end{gathered}
$$

By using assumption (i), we have

$$
\|u-P u\|_{X} \leqq k_{1}\|N u\|_{Z} \leqq k_{1}\left[\langle N u, \xi u\rangle+\alpha\|u\|_{X}+\beta\right]
$$

where $k_{1}$ is the norm of the continuous linear operator $K(I-Q)$.

Now, if $u$ satisfies (2.6), $L u=\lambda N u+(1-\lambda) \Phi u$ and therefore, from (2.7) and (ii.1),

$$
\|u-P u\|_{x} \leqq k_{1} \alpha\|u\|_{X}+k_{1} \beta
$$


Also, it follows from hypothesis (ii.2) that for all $k, 1 \leqq k \leqq m$, there exists $x_{k} \in G$ such that $\left|u_{k}\left(x_{k}\right)\right|<r \phi_{k}\left(x_{k}\right)$. In fact, if it is not the case, then $\left|u_{k}(x)\right| \geqq r \phi_{k}(x), \forall x \in G$, for some $k$, $1 \leqq k \leqq m$ and, by assumption (ii. 2$),(Q N)_{j}(u)(\Phi)_{j}(u) \geqq 0$ for some $j, 1 \leqq j \leqq m$. As

$$
(Q N)_{j}(u)(\Phi)_{j}(u)=\frac{-\lambda}{1-\lambda}(Q N)_{j}(u)(Q N)_{j}(u) \leqq 0
$$

we have $(Q N)_{j}(u)=(\Phi)_{j}(u)=0$ and this contradicts (ii.2) $\left((\Phi)_{j}(u)\right.$ is not zero).

Therefore

$$
\begin{array}{r}
\left|(P u)_{k}(x)\right| \phi_{k}\left(x_{k}\right) \leqq\left|\int_{G} u_{k}(x) \phi_{k}(x) d x\right| \phi_{k}(x) \phi_{k}\left(x_{k}\right) \\
\leqq k_{2}\left|(P u)_{k}\left(x_{k}\right)\right| \leqq k_{2}\left(\left|u_{k}\left(x_{k}\right)\right|+\left|(I-P)_{k}(u)\left(x_{k}\right)\right|\right) \\
\leqq k_{2} r \phi_{k}\left(x_{k}\right)+c\|(I-P) u\|_{X} \phi_{k}\left(x_{k}\right), \quad \forall x \in G .
\end{array}
$$

Thus

$$
\sup _{x \in G}\left|(P u)_{k}(x)\right| \leqq k_{2} r+c\|(I-P) u\|_{x}
$$

and since all the norms in a finite dimensional space $(\operatorname{Im} P)$ are equivalent, we deduce

$$
\|P u\|_{x} \leqq k_{3}+k_{3} c\|(I-P) u\|_{x}
$$

for a certain $k_{3}>0$. Therefore

$$
\begin{aligned}
\|u\|_{X} & \leqq\|P u\|_{X}+\|u-P u\|_{X} \\
& \leqq k_{1} \alpha\left(1+k_{3} c\right)\|u\|_{x}+\left(k_{1} \beta+k_{3}+k_{1} k_{3} c \beta\right) .
\end{aligned}
$$

Then, if $k_{1} \alpha\left(1+k_{3} c\right)<1$, we obtain

$$
\|u\|_{x} \leqq \frac{k_{1} \beta+k_{3}+k_{1} k_{3} c \beta}{1-k_{1} \alpha\left(1+k_{3} c\right)} \equiv r_{0}
$$

Lastly, if $\lambda=0$ in (2.5), then $u-P u=J \Phi u$ and therefore, $u=P u, \Phi u=0$. As $u=P u$, $u \in \operatorname{ker} L$, i.e., $u(x)=\left(a_{1} \phi_{1}(x), \ldots, a_{m} \phi_{m}(x)\right)$ for some $\left(a_{1}, \ldots, a_{m}\right) \in R^{m}$. From (ii. 2$),\left|a_{k}\right|<r$ for all $1 \leqq k \leqq m$.

Taking $\Omega=B_{X}\left(r_{1}\right), r_{1}>\max \left\{r_{0}, r\right\}$, the proof is finished.

Remarks. (1) As we shall see, the mappings $L$ that satisfy $(\mathrm{H})$ include a great class of linear elliptic operators together with suitable boundary conditions.

(2) Theorem 2.1 is very general and we may take different functions $\Phi$ to obtain some 
existence theorems for equation (2.1). In view of the applications we consider only two cases. We begin with a corollary which is related to a result obtained by the author and Ortega [5] and the author and Martinez-Amores [4].

Corollary 2.1. Assume $(\mathrm{H})$ holds and that:

(a) Conditions (i) and (ii.3) of Theorem 2.1 hold.

(b) $\langle L u, \xi u\rangle \leqq 0$ for all $u \in \operatorname{dom} L$.

(c) There exists $r>0$ such that for all $u \in X, u=\left(u_{1}, \ldots, u_{m}\right)$ with $\left|u_{k}(x)\right| \geqq r \phi_{k}(x), \forall x \in G$ for some $k, 1 \leqq k \leqq m$, one has $Q N u \neq 0$.

Then, if $\propto$ is sufficiently small, equation (2.1) has at least one solution.

Proof. Take $\Phi=Q N$ in Theorem 2.1 .

Corollary 2.2. Assume $(\mathrm{H})$ holds and that $N$ is uniformly bounded, i.e., $\|N u\|_{Z} \leqq \beta$ for every $u \in X$. Also, we suppose that

$$
\operatorname{Im} L=\left\{z=\left(z_{1}, \ldots, z_{m}\right) \in Z: \int_{G} z_{k}(x) \psi_{k}(x) d x=0,1 \leqq k \leqq m\right\}
$$

where the functions $\psi_{k}: \bar{G} \rightarrow R, 1 \leqq k \leqq m$, are continuous, positive on $G$ and normalized $\left(\int_{G} \psi_{k}^{2}(x) d x=1\right)$.

Then, if hypothesis

(a) There is $r>0$ such that for all $u \in X, u=\left(u_{1}, \ldots, u_{m}\right)$ with $\left|u_{k}(x)\right| \geqq r \phi_{k}(x) \forall x \in G$, for some $k, 1 \leqq k \leqq m$, one has sign $u_{k}(Q N u)_{\sigma(k)} \leqq 0$ (where $\sigma$ is a permutation of the indices $1, \ldots, m)$, is satisfied, equation (2.1) has at least one solution.

Proof. $\left(\mathrm{H}_{1}\right)$ allows us to take $Q: Z \rightarrow Z$ defined by

$$
(Q z)_{k}(x)=\psi_{k}(x) \int_{G} z_{k}(x) \psi_{k}(x) d x, \quad 1 \leqq k \leqq m
$$

Now, take in Theorem $2.1 \Phi: X \rightarrow \operatorname{Im} Q$ defined by $(\Phi u)_{k}=-(Q u)_{\sigma-1(k)}, 1 \leqq k \leqq m$. Trivially hypotheses (i), (ii.1) are verified with $\xi \equiv 0$.

On the other hand, if $\left|u_{k}(x)\right| \geqq r \phi_{k}(x) \forall x \in G$, then either $u_{k}(x) \geqq r \phi_{k}(x) \forall x \in G$ or $u_{k}(x) \leqq-r \phi_{k}(x) \forall x \in G$. In the first case by taking $j=\sigma(k)$ in (ii.2), we have that (ii.2) becomes

$$
\begin{aligned}
(Q N u)_{\sigma(k)}(\Phi u)_{\sigma(k)} & =(Q N u)_{\sigma(k)}(-Q u)_{k} \\
& =-(Q N u)_{\sigma(k)} \int_{G} u_{k}(x) \psi_{k}(x) d x \psi_{k}(x) \geqq 0
\end{aligned}
$$

This implies that $(Q N u)_{\sigma(k)} \leqq 0$.

Analogously if $u_{k}(x) \leqq-r \phi_{k}(x) \forall x \in G$, one obtains $(Q N u)_{\sigma(k)} \geqq 0$. Therefore (a) of 
Corollary 2.2 becomes the same as (ii.2) of Theorem 2.1. Also

$$
d_{B}\left(\left.J \Phi\right|_{\text {ker } L}, B_{X}(s) \cap \operatorname{ker} L, 0\right) \neq 0 \Leftrightarrow d_{B}\left(F, B_{R^{m}}(s), 0\right) \neq 0
$$

where $F: R^{m} \rightarrow R^{m}$ is defined by

$$
(F(a))_{k}=a_{\sigma-1(k)} \int_{G} \phi_{k}(x) \psi_{k}(x) d x, \quad 1 \leqq k \leqq m .
$$

As $(F(a))_{k}=a_{\sigma-1_{(k)}} c_{k}, c_{k}>0,1 \leqq k \leqq m$, the last degree is different from zero and the corollary is proved.

Let us think now in the non-resonance case, i.e., $\operatorname{ker} L$ is trivial. Then $P=0, Q=0$ and $K=L^{-1}$. Assuming that $L^{-1}$ is continuous and that $L^{-1} N$ is compact on bounded subsets of $X$, we have the following result. (The proof is very similar to the proof of Theorem 2.1.)

Theorem 2.2 ([5]). Let us suppose that there exists a mapping $\xi: X \rightarrow Z^{*}$ verifying $\langle L u, \xi u\rangle \leqq 0$ for all $u \in \operatorname{dom} L$ and that $\|N u\|_{Z} \leqq\langle N u, \xi u\rangle+\alpha\|u\|_{X}+\beta$ for all $u \in X$ and constants $\alpha \geqq 0, \beta \geqq 0$. Then, if $\alpha$ is sufficiently small, equation (2.1) has at least one solution.

\section{Applications}

In this section we use the results of the previous one to study some non-linear boundary value problems for elliptic systems.

Let $G \subset R^{n}$ be a bounded domain, $p$ a natural number, $p>n$, and $\alpha \in R$ such that $0<\alpha<1-n / p$. We shall deal with the classical solvability of non-linear elliptic boundary value problems of the type

$$
\begin{gathered}
A_{k} u_{k}-\lambda_{1}^{k} u_{k}=g_{k}\left(x, u_{1}, \ldots, u_{m}\right), \quad x \in G, \quad 1 \leqq k \leqq m \\
B_{k} u_{k}=0, \quad x \in \partial G, \quad 1 \leqq k \leqq m
\end{gathered}
$$

where $A_{k}$ is a second-order uniformly elliptic operator

$$
A_{k}=-\sum_{i, j=1}^{n} a_{i j}^{k}(x) \frac{\partial^{2}}{\partial x_{i} \partial x_{j}}+\sum_{i=1}^{n} a_{i}^{k}(x) \frac{\partial}{\partial x_{i}}, \quad 1 \leqq k \leqq m
$$

with $a_{i j}^{k}=a_{j i}^{k}$. The boundary $\partial G$ and the coefficients of $A_{k}, 1 \leqq k \leqq m$, are supposed to be smooth, let us say $a_{i j}^{k}, a_{i}^{k} \in C^{2, a}(\bar{G}), \partial G \in C^{2, a}$, with

$$
\mu_{k} \sum_{i=1}^{n} \xi_{i}^{2} \leqq \sum_{i, j=1}^{n} a_{i j}^{k}(x) \xi_{i} \xi_{j}, \quad \forall x \in G, \quad \forall \xi_{i} \in R, \quad 1 \leqq i \leqq n
$$

where $\mu_{k}>0$ is a constant and $1 \leqq k \leqq m$. 
We restrict ourselves to linear homogeneous boundary conditions $B_{k} u_{k}=0$, which will be either Dirichlet type $\left(u_{k}=0\right.$ on $\left.\partial G\right)$ or Neumann type $\partial u_{k} / \partial v_{A_{k}}=0$ on $\partial G$, where $v_{A_{k}}$ is the unit outer conormal vector field on $\partial G$ and $\partial / \partial v_{A_{k}}$ is the outer conormal derivative on $\partial G$ ).

For the sake of simplicity we shall assume that the functions $g_{k}: \bar{G} \times R^{m} \rightarrow R, 1 \leqq k \leqq m$, are continuously differentiable.

From the above hypotheses it follows that the problem

$$
\begin{array}{ll}
A_{k} u_{k}=\lambda u_{k}, & x \in G \\
B_{k} u_{k}=0, & x \in \partial G
\end{array}
$$

has a unique normalized positive solution $\phi_{k}$ on $G$ which corresponds to a simple real eigenvalue $\lambda_{1}^{k}$ ( $\lambda_{1}^{k}=0$ and $\phi_{k}=$ constant under the Neumann boundary condition).

Taking into account the properties of $\phi_{k}$ it is possible to prove the following statement ([10]): Given any bounded set $X_{0}$ of functions in $C^{1}(\bar{G}, R)$, if $B_{k} u_{k}=0$, for all $u_{k} \in X_{0}$, then

$$
\alpha \phi_{k}(x)<u_{k}(x)<\beta \phi_{k}(x) \text { on } G
$$

for some constants $\alpha, \beta$ independent of $u_{k}$.

Now, the following important result is easily established:

Lemma 3.1. There exists $c_{k}>0$ such that

$$
\left|u_{k}(x)\right| \leqq c_{k} \phi_{k}(x)\left\|u_{k}\right\|_{1} \quad \text { on } \bar{G}
$$

where

$$
\left\|u_{k}\right\|_{1}=\sum_{|\beta| \leqq 1} \sup _{x \in G}\left|D^{\beta} u_{k}(x)\right|
$$

for all $u_{k} \in C^{1}(\bar{G}, R)$ satisfying $B_{k} u_{k}=0$.

Proof. Take

$$
X_{0}=\left\{\frac{u_{k}}{\left\|u_{k}\right\|_{1}}: u_{k} \in C^{1}(\bar{G}, R), u_{k} \neq 0, B_{k} u_{k}=0\right\}
$$

in the inequality (3.3).

The adjoint problem to $A_{k} u_{k}-\lambda_{1}^{k} u_{k}=0, x \in G ; B_{k} u_{k}=0, x \in \partial G$, has also a onedimensional space of solutions generated by a normalized positive function $\psi_{k}$ on $G$ and such that if $f \in C^{0, \alpha}(\vec{G}, R)$, the problem

$$
\begin{array}{ll}
A_{k} u_{k}-\lambda_{1}^{k} u_{k}=f, & x \in G \\
B_{k} u_{k}=0, & x \in \partial G
\end{array}
$$


has a solution $u_{k} \in C^{2, \alpha}(\bar{G}, R)$ if and only if $\int_{G} f(x) \psi_{k}(x) d x=0$ (also, $\psi_{k}=$ constant under the Neumann boundary condition).

We put

$$
X=\left\{u \in C^{1, \alpha}(\bar{G}, R) \times \stackrel{(m)}{\cdots} \times C^{1, \alpha}(\bar{G}, R), u=\left(u_{1}, \ldots, u_{m}\right): B_{k} u_{k}=0,1 \leqq k \leqq m\right\}
$$

with the norm

$$
\|u\|_{1, \alpha}=\left(\sum_{k=1}^{m}\left\|u_{k}\right\|_{1, \alpha}^{p}\right)^{1 / p}
$$

where for each $k, 1 \leqq k \leqq m$,

$$
\left\|u_{k}\right\|_{1, \alpha}=\max _{|\beta| \leqq 1} \sup _{x \in G}\left|D^{\beta} u_{k}(x)\right|+\max _{|\beta| \leqq 1} \sup _{\substack{x, y \in G \\ x \neq y}} \frac{\left|D^{\beta} u_{k}(x)-D^{\beta} u_{k}(y)\right|}{|x-y|^{\alpha}}
$$

and

$$
Z=\left\{z \in C^{0, \alpha}(\bar{G}, R) \times \stackrel{(m)}{\cdots} \times C^{0, \alpha}(\bar{G}, R)\right\}
$$

with the norm

$$
\|z\|_{p}=\left(\sum_{k=1}^{m}\left\|z_{k}\right\|_{p}^{p}\right)^{1 / p}, \quad z=\left(z_{1}, \ldots, z_{m}\right)
$$

where for each $k, 1 \leqq k \leqq m$,

$$
\left\|z_{k}\right\|_{p}=\left(\int_{G}\left|z_{k}(x)\right|^{p} d x\right)^{1 / p}
$$

If we define $L: \operatorname{dom} L \subset X \rightarrow Z$ by

$$
\operatorname{dom} L=\left\{u \in X: u \in C^{2, \alpha}(\bar{G}, R) \times \stackrel{(m)}{\cdots} \times C^{2, \alpha}(\bar{G}, R)\right\}
$$

$(L u)_{k}=A_{k} u_{k}-\lambda_{1}^{k} u_{k}$, and $N: X \rightarrow Z$ by $(N u)_{k}(x)=g_{k}\left(x, u_{1}(x), \ldots, u_{m}(x)\right)$, for all $u \in X$, and $x \in G$, then our problem (3.1) is equivalent to solving the operator equation

$$
L u=N u
$$


Now $\operatorname{ker} L=\left\{u \in X: u=\left(a_{1} \phi_{1}, \ldots, a_{m} \phi_{m}\right),\left(a_{1}, \ldots, a_{m}\right) \in R^{m}\right\}$

$$
\operatorname{Im} L=\left\{z \in Z, z=\left(z_{1}, \ldots, z_{m}\right): \int_{G} z_{k}(x) \psi_{k}(x) d x=0,1 \leqq k \leqq m\right\}
$$

and $L$ is a linear Fredholm mapping of index zero.

It is trivial that $P: X \rightarrow X,(P u)_{k}(x)=\phi_{k}(x) \int_{G} u_{k}(x) \phi_{k}(x) d x, 1 \leqq k \leqq m$ and $Q: Z \rightarrow Z$, $(Q z)_{k}(x)=\psi_{k}(x) \int_{G} z_{k}(x) \psi_{k}(x) d x, 1 \leqq k \leqq m$, are projectors such that $\operatorname{Im} P=\operatorname{ker} L, \operatorname{Im} L=\operatorname{ker} Q$ and if $K$ is the generalized inverse of $L(K: \operatorname{Im} L \rightarrow \operatorname{dom} L n \operatorname{ker} P)$, there exists $c(p)>0$ such that

$$
\left\|(K f)_{k}\right\|_{W^{2 . p(\bar{G}, R)}} \leqq c(p)\left\|f_{k}\right\|_{p}, \quad 1 \leqq k \leqq m
$$

(see [2]). As the inclusion $W^{2, p}(\bar{G}, R) \hookrightarrow C^{1, \alpha}(\bar{G}, R)$ is compact $([1]), K:\left(\operatorname{Im} L,\|\cdot\|_{p}\right) \rightarrow$ $\left(X,\|\cdot\|_{1, \alpha}\right)$ is compact.

Now, we are in position to apply the results of the previous section to system (3.1). Firstly we assume that $g_{k}, 1 \leqq k \leqq m$, are bounded.

Theorem 3.1. Suppose that:

(i) There is $r>0$ such that for all $u \in X, u=\left(u_{1}, \ldots, u_{m}\right)$ with $\left|u_{k}(x)\right| \geqq r \phi_{k}(x), \forall x \in G$, for some $k, 1 \leqq k \leqq m$, one has

$$
\operatorname{sign} u_{k} \int_{G}\left(g_{\sigma(k)}\left(x, u_{1}(x), \ldots, u_{m}(x)\right)\right) \psi_{k}(x) d x \leqq 0
$$

where $\sigma$ is a permutation of the indices $1, \ldots, m$.

Then, (3.1) has at least one solution.

The proof is trivial from Corollary 2.2 .

Remarks. (1) Theorem (3.1) is still true if the inequality in hypothesis (i) is reversed.

(2) Let $m=1$ and $g=g(x, u)$ satisfying:

(i') There is $r>0$ such that $\int_{G} g(x, u(x)) \psi_{1}(x) d x \cdot \int_{G} g(x, v(x)) \psi_{1}(x) d x<0$ for all $u, v \in X$ with $u(x) \geqq r \phi_{1}(x), v(x) \leqq-r \phi_{1}(x), \forall x \in G$.

Then, assumption (i) of Theorem 3.1 is satisfied. In fact, if $u_{0} \in X$ verifies $u_{0}(x) \geqq r \phi_{1}(x), \forall x \in G$ and $\int_{G} g\left(x, u_{0}(x)\right) \psi_{1}(x) d x<0$, one has $\int_{G} g(x, u(x)) \psi_{1}(x) d x<0$ for all $u \in X$ verifying $u(x) \geqq r \phi_{1}(x), \forall x \in G$. (The set $A=\left\{u \in X: u(x) \geqq r \phi_{1}(x), \forall x \in G\right\}$ is connected and the mapping from $X$ into $R$ defined by $u \rightarrow \int_{G} g(x, u(x)) \psi_{1}(x) d x$ is continuous.) Hence, $\int_{G} g(x, v(x)) \psi_{1}(x) d x>0$ for all $v \in X$ verifying $v(x) \leqq-r \phi_{1}(x), \forall x \in G$, and therefore (i) of Theorem 3.1 is verified with a strict inequality.

If $\int_{G} g\left(x, u_{0}(x)\right) \psi_{1}(x) d x>0$, we should consider the previous remark.

Hypothesis (i') has been considered by de Figueiredo and Ni [7]. Thus, our Theorem 3.1 generalizes, yet in the scalar case, the main result of these authors. 


\section{A. CAÑADA}

(3) Let $g_{k}, \quad 1 \leqq k \leqq m$, be such that $g_{\sigma(k)}\left(x, u_{1}, \ldots, u_{m}\right) \cdot u_{k} \leqq 0$ for all $x \in G$, $\left(u_{1}, \ldots, u_{m}\right) \in R^{m}, 1 \leqq k \leqq m$. Then, assumption (i) of Theorem 3.1 is satisfied. (The inequality can be reversed.)

(4) Let $g_{k}, 1 \leqq k \leqq m$, satisfy the following conditions:

$(\mathrm{A}+)$ There exist $S_{1} \in R$ and a bounded continuous mapping $g_{+}^{k}: \bar{G} \times R^{m} \rightarrow R, 1 \leqq k \leqq m$, such that for all $u \in X, u=\left(u_{1}, \ldots, u_{m}\right)$ with $u_{k}(x)>S_{1} \phi_{k}(x), \quad \forall x \in G$, one has $g_{k}\left(x, u_{1}(x), \ldots, u_{m}(x)\right) \leqq g_{+}^{k}\left(x, u_{1}(x), \ldots, u_{m}(x)\right), \forall x \in G$ and

$$
\int_{G} g_{+}^{k}\left(x, u_{1}(x), \ldots, u_{m}(x)\right) \psi_{k}(x) d x \leqq 0
$$

(A-) There exist $S_{2} \in R$ and a bounded continuous mapping $g_{-}^{k}: \bar{G} \times R^{m} \rightarrow R, 1 \leqq k \leqq m$, such that for all $u \in X, u=\left(u_{1}, \ldots, u_{m}\right)$ with $u_{k}(x)<S_{2} \phi_{k}(x), \forall x \in G$, one has $g_{k}\left(x, u_{1}(x), \ldots, u_{m}(x)\right) \geqq g^{k}\left(x, u_{1}(x), \ldots, u_{m}(x)\right), \forall x \in G$ and

$$
\int_{G} g_{-}^{k}\left(x, u_{1}(x), \ldots, u_{m}(x)\right) \psi_{k}(x) d x \geqq 0 .
$$

Then, condition (i) of Theorem 3.1 is again satisfied (see [6], where the details are given for the ordinary case).

Therefore, Theorem 3.1 extends and generalizes, for systems with bounded nonlinearities, the results given by Kazdan and Warner [10] in the scalar case.

Example 1. The B.V.P.

$$
\begin{gathered}
-\Delta u_{1}-\lambda_{1} u_{1}=g_{1}\left(u_{2}\right)+f_{1}(x), \quad x \in G \\
-\Delta u_{2}-\lambda_{1} u_{2}=g_{2}\left(u_{1}\right)+f_{2}(x), \quad x \in G \\
u_{1}(x)=u_{2}(x)=0, \quad x \in \partial G
\end{gathered}
$$

has at least one solution assuming that $\int_{G} f_{i}(x) \psi(x) d x=0,1 \leqq i \leqq 2$, and $g_{1}\left(u_{2}\right) u_{2} \geqq 0$, $g_{2}\left(u_{1}\right) u_{1} \geqq 0$.

This example cannot be studied from the results mentioned in the previous remarks, because it is a vector problem. Yet in the scalar case, we may consider the B.V.P.

$$
\begin{aligned}
-\Delta u-\lambda_{1} u & =h(u)+f(x), \quad x \in G \\
u & =0, \quad x \in \partial G
\end{aligned}
$$

where $h: R \rightarrow R$ is of class $C^{1}$ and bounded, $f: \bar{G} \rightarrow R$ is continuous and $\int_{G} f(x) \psi(x) d x=0$.

If $h(u) \cdot u>0$ for all $u \in R$, all conditions of Theorem 3.1 are satisfied (with the reversed inequality) and (3.6) has a solution. However, (3.6) does not satisfy $(\mathrm{A}+)$ and $(\mathrm{A}-)$. In fact, if $g(x, u)=h(u)+f(x)$ and $u \in X$ is such that $u(x)>S_{1} \phi(x), \forall x \in G$ with $u(x)>0$ 
$\forall x \in G$, one obtains

$$
0 \geqq \int_{G} g_{+}(x, u(x)) \psi(x) d x \geqq \int_{G} g(x, u(x)) \psi(x) d x=\int_{G} h(u(x)) \psi(x) d x
$$

which is a contradiction. Also $(\mathrm{A}-)$ is not satisfied.

Let us consider now the case where $g_{k}, 1 \leqq k \leqq m$, can have a growth of some superlinear type. First we need a lemma.

Lemma 3.2. Let $A$ be a second-order uniform operator of the form

$$
A=-\sum_{i=1}^{n} \frac{\partial}{\partial x_{i}}\left(\sum_{j=1}^{n} a_{i j}(x) \frac{\partial}{\partial x_{j}}\right)
$$

where the coefficients of $A$ satisfy the same regularity assumptions considered at the beginning of this section for $A_{k}$ and let $u \in C^{2, \alpha}(\bar{G}, R)$ be such that $\partial u / \partial v_{A}=0$ on $\partial G$. Then, if $w \in C^{1}(R, R)$ verifies $w^{\prime}(t) \leqq 0$ for all $t \in R$, we have that $w \circ u \in C^{0, \alpha}(\vec{G}, R)$ and

$$
\int_{G} A u(x) w(u(x)) d x \leqq 0
$$

Proof. It is trivial that $w \circ u \in C^{1}(\bar{G}, R)$. But as $\partial G \in C^{2, \alpha}, C^{1}(\bar{G}) \hookrightarrow C^{0, \alpha}(\bar{G})$ (see $[8]$ ) and then $w \circ u \in C^{0, \alpha}(\bar{G})$.

On the other hand, by using the Green's formula ([9, p. 69]), we deduce ( $v_{i}$ denotes the $i$ th component of the unit vector of the outward normal $v$ to $\partial G$ ),

$$
\begin{aligned}
\int_{G} A u(x) w(u(x)) d x= & -\sum_{i=1}^{n} \int_{G} \frac{\partial}{\partial x_{i}}\left(\sum_{j=1}^{n} a_{i j}(x) \frac{\partial u(x)}{\partial x_{j}}\right) w(u(x)) d x \\
= & -\sum_{i=1}^{n}\left[-\int_{G} w^{\prime}(u(x)) \frac{\partial u(x)}{\partial x_{i}}\left(\sum_{j=1}^{n} a_{i j}(x) \frac{\partial u(x)}{\partial x_{j}}\right) d x\right. \\
& +\int_{\partial G} w(u(x))\left(\sum_{j=1}^{n} a_{i j}(x) \frac{\partial u(x)}{\partial x_{j}} v_{i}(x) d S\right] \\
= & \int_{G} w^{\prime}(u(x)) \sum_{i, j=1}^{n} a_{i j}(x) \frac{\partial u(x)}{\partial x_{i}} \frac{\partial u(x)}{\partial x_{j}} d x \\
& -\int_{\partial G} w(u(x)) \sum_{i, j=1}^{n} a_{i j}(x) \frac{\partial u(x)}{\partial x_{j}} v_{i}(x) d s \\
\leqq & \int_{G} w^{\prime}(u(x)) \mu \sum_{i=1}^{n}\left(\frac{\partial u(x)}{\partial x_{i}}\right)^{2} d x-\int_{\partial G} w(u(x)) \frac{\partial u(x)}{\partial v_{A}} d s \leqq 0 .
\end{aligned}
$$

When the boundary conditions are of Dirichlet type, an analogous result may be proved by the same way. 
Lemma 3.3. Let $u \in C^{2, a}(\bar{G}, R)$ be such that $u=0$ on $\partial G$ and $w \in C^{1}(R, R)$ satisfying $w^{\prime}(t) \leqq 0$ for all $t \in R$ and $w(0)=0$. Then $w \circ u \in C^{0, \alpha}(\bar{G}, R)$ and

$$
\int_{G} A u(x) w(u(x)) d x \leqq 0
$$

Remarks. (1) A similar inequality to (3.7), (3.8) has been proved by Brezis and Strauss [3] for elliptic B.V.P. in $L^{1}$.

(2) It is not possible to obtain an analogous result to the previous lemmas for problems whose linear part is of the form $A u(x)-\lambda_{1} u(x), x \in G, u(x)=0, x \in \partial G$. (Take the Dirichlet problem for $n=1, G=(0, \pi)$. Then, $A u(x)-\lambda_{1} u(x)=-u^{\prime \prime}(x)-u(x), u(0)=$ $u(\pi)=0$, and $w: R \rightarrow R$ defined by

$$
w(t)=\left\{\begin{array}{l}
0 \text { if } t<0 \\
-t^{2} \text { if } t \geqq 0
\end{array}\right.
$$

Then, if $u(x)=-\left(x^{2}-\pi x\right)$, we have

$$
\left.\int_{G} A u(x) w(u(x)) d x=\int_{0}^{\pi}-\left(x^{2}-\pi x\right)\left[-2(2 x-\pi)^{2}+\left(x^{2}-\pi x\right)^{2}\right] d x=\pi^{5}\left(\frac{\pi^{2}}{140}-\frac{1}{15}\right)>0\right) .
$$

Theorem 3.2. Let us suppose that:

(i) There exists a mapping $w: R^{m} \rightarrow R^{m}$ of class $C^{1}, w\left(u_{1}, \ldots, u_{m}\right)=\left(w_{1}\left(u_{1}\right), \ldots, w_{m}\left(u_{m}\right)\right)$ such that $w_{k}: R \rightarrow R, 1 \leqq k \leqq m$, verifies $w_{k}^{\prime}(t) \leqq 0$ for all $t \in R$, and constants $\alpha_{1}^{\prime} \geqq 0, \beta_{1}^{\prime} \geqq 0$, satisfying:

$$
\sum_{k=1}^{m}\left|g_{k}\left(x, u_{1}, \ldots, u_{m}\right)\right|^{p} \leqq\left(w\left(u_{1}, \ldots, u_{m}\right), g\left(x, u_{1}, \ldots, u_{m}\right)\right)+\alpha_{1}^{\prime}|u|+\beta_{1}^{\prime}
$$

for all $(x, u)=\left(x, u_{1}, \ldots, u_{m}\right) \in \bar{G} \times R^{m}$ (where $(\cdot, \cdot)$ denotes the usual inner product in $\left.R^{m}\right)$, $g=\left(g_{1}, \ldots, g_{m}\right)$

(ii) There exists $r>0$ such that for all $u \in X, u=\left(u_{1}, \ldots, u_{m}\right)$ with $\left|u_{k}(x)\right| \geqq r$ for each $x \in G$ and some $k, 1 \leqq k \leqq m$, one has $\int_{G} g\left(x, u_{1}(x), \ldots, u_{m}(x)\right) d x \neq 0$.

(iii) $d_{B}\left(F, B_{R^{m}}(s), 0\right) \neq 0$ for every $s \geqq r$, where $F: R^{m} \rightarrow R^{m}$ is defined by $F(c)=\int_{G} g(x, c) d x$.

Then, the B.V.P. (3.1), where each $A_{k}$ has the form

$$
A_{k}=-\sum_{i=1}^{n} \frac{\partial}{\partial x_{i}}\left(\sum_{j=1}^{n} a_{i j}^{k}(x) \frac{\partial}{\partial x_{j}}\right), \quad 1 \leqq k \leqq m
$$

and $B_{k} u_{k}=\partial u_{k} / \partial v_{A_{k}}, 1 \leqq k \leqq m$, has at least one solution provided $\alpha_{1}^{\prime}$ is sufficiently small. 
Proof. Take in Corollary $2.1 \xi: X \rightarrow Z^{*}$ defined by

$$
(\xi u)(z)=\sum_{k=1}^{m} \int_{G} w_{k}\left(u_{k}(x)\right) z_{k}(x) d x
$$

It is obvious that $(\mathrm{H})$ is satisfied with $\phi_{k}=1 / \operatorname{vol}(G), 1 \leqq k \leqq m$. On the other hand, using (i) we have

$$
\|N u\|_{p}^{p}=\sum_{k=1}^{m}\left\|(N u)_{k}\right\|_{p}^{p}=\sum_{k=1}^{m} \int_{G}\left|g_{k}\left(x, u_{1}, \ldots, u_{m}\right)\right|^{p} d x \leqq\langle N u, \xi u\rangle+\alpha_{1}^{\prime \prime}\|u\|_{1, \alpha}+\beta_{1}^{\prime \prime}
$$

for all $u \in X$. Since there is $\gamma>0$ such that $t \leqq t^{p}+\gamma$ for all $t \geqq 0$ we have condition (i) of Theorem 2.1.

Also, taking into account Lemma 3.2 we have hypothesis (b) of Corollary 2.1.

Lastly, condition (ii.3) of Theorem 2.1 and (c) of Corollary 2.1 are respectively the same as (iii) and (ii) in Theorem 3.2 .

Then all conditions of Corollary 2.1 are satisfied and the theorem is proved.

Example 2. Let us consider the B.V.P.

$$
\begin{array}{ll}
-\Delta u_{1}=-e^{u_{1}}+h_{1}\left(x, u_{1}, u_{2}\right)+f_{1}(x), & x \in G \\
-\Delta u_{2}=-e^{u_{2}}+h_{2}\left(x, u_{1}, u_{2}\right)+f_{2}(x), & x \in G \\
\frac{\partial u_{1}}{\partial v}=\frac{\partial u_{2}}{\partial v}=0, & x \in \partial G,
\end{array}
$$

where $f_{i}: \bar{G} \rightarrow R, h_{i}: \bar{G} \times R^{2} \rightarrow R$ are of class $C^{1}, h_{i}(x, u) \leqq 0$ for all $x \in \bar{G}, u \in R^{2}$ and $h_{i}$ are bounded and satisfy $\lim _{\left|u_{1}\right|+\left|u_{2}\right| \rightarrow \infty} h_{i}\left(x, u_{1}, u_{2}\right)=0$ uniformly in $x \in \bar{G}, 1 \leqq i \leqq 2$.

Then, (3.9) has one solution if, and only if, $\int_{G} f_{i}(x) d x>0, i=1,2$.

The above condition is clearly necessary and is also sufficient. In fact, let $p>n$ be an odd number and $w_{i}: R \rightarrow R, 1 \leqq i \leqq 2$, defined by

$$
w_{i}(t)= \begin{cases}0 & \text { if } t \leqq 0 \\ \left(-e^{t}+t+1\right)^{p} & \text { if } t>0 .\end{cases}
$$

Then

$$
\begin{array}{rlrl}
\left|g_{i}\left(x, u_{1}, u_{2}\right)\right|^{p} & =\left|-e^{u_{i}}+h_{i}\left(x, u_{1}, u_{2}\right)+f_{i}(x)\right|^{p} \leqq \beta & & \text { if } u_{i} \leqq 0 \\
\left|g_{i}\left(x, u_{1}, u_{2}\right)\right|^{p} & =\left|-e^{u_{i}}+h_{i}\left(x, u_{1}, u_{2}\right)+f_{i}(x)\right|^{p} & \\
& \leqq\left(-e^{u_{i}}+u_{i}+1\right)^{p}\left(-e^{u_{i}}+h_{i}\left(x, u_{1}, u_{2}\right)+f_{i}(x)\right)+\beta^{\prime} & & \text { if } \quad u_{i}>0
\end{array}
$$

and therefore, (i) of Theorem 3.2 is satisfied with $\alpha_{1}^{\prime}=0$. 
Hypothesis (ii) of this theorem is trivially verified. Also $F: R^{2} \rightarrow R^{2}$ is now defined by $F\left(c_{1}, c_{2}\right)=\left(F_{1}\left(c_{1}, c_{2}\right), F_{2}\left(c_{1}, c_{2}\right)\right)$, where

$$
\begin{aligned}
& F_{1}\left(c_{1}, c_{2}\right)=-e^{c_{1}}+(1 / \operatorname{vol}(G))\left[\int_{G} h_{1}\left(x, c_{1}, c_{2}\right) d x+\int_{G} f_{1}(x) d x\right] \\
& F_{2}\left(c_{1}, c_{2}\right)=-e^{c_{2}}+(1 / \operatorname{vol}(G))\left[\int_{G} h_{2}\left(x, c_{1}, c_{2}\right) d x+\int_{G} f_{2}(x) d x\right] .
\end{aligned}
$$

It is easily proved that there exists $r_{1}>0$ such that $F_{i}\left(c_{1}, c_{2}\right) c_{i} \leqq 0$, with $\left|c_{i}\right| \geqq r_{1}, i=1,2$. Then, taking into account the Poincaré-Bohl theorem [14], (iii) of Theorem 3.2 is also verified.

Remarks, (1) If $w_{k}=0,1 \leqq k \leqq m$, condition (i) may be substituted by

$$
\left|g_{k}\left(x, u_{1}, \ldots, u_{m}\right)\right| \leqq \alpha_{k}^{\prime}\left|u_{k}\right|+\beta_{k}^{\prime}, \quad 1 \leqq k \leqq m .
$$

Therefore, Theorem 3.2 generalizes the main result obtained by Mawhin in [12], not only with respect to the growth of the non-linear term but also because we consider systems of equations.

(2) In the scalar case, B.V.P. of type (3.9) may be studied using the method of upper and lower solutions [10], but the use of this method in the case of vector problems is very restrictive. (We know that either certain monotone properties on the components $g_{k}$ of the non-linearity $g$ are required or a stronger definition of upper and lower solutions is needed.)

Finally and as an application of Theorem 2.2 we can study the existence of solutions of the Dirichlet problem

$$
\begin{array}{ll}
A_{k} u_{k}=g_{k}\left(x, u_{1}, \ldots, u_{m}\right), & x \in G, \quad 1 \leqq k \leqq m \\
u_{k}=0, & x \in \partial G, \quad 1 \leqq k \leqq m .
\end{array}
$$

Theorem 3.3. Let us assume that there exists a mapping $w: R^{m} \rightarrow R^{m}$ verifying the hypotheses of the previous theorem and, moreover, $w_{k}(0)=0,1 \leqq k \leqq m$. Then, if (i) of Theorem 3.2 is satisfied, equation (3.10) has at least one solution provided $\alpha_{1}^{\prime}$ is sufficiently small.

Example 3. The B.V.P.

$$
\begin{array}{ll}
-\Delta u_{1}+\lambda u_{1}=-u_{1}^{p_{1}}+h_{1}\left(x, u_{1}, u_{2}\right), & x \in G \\
-\Delta u_{2}+\mu u_{2}=-u_{2}^{p_{2}}+h_{2}\left(x, u_{1}, u_{2}\right), & x \in G \\
u_{1}=u_{2}=0, & x \in \partial G
\end{array}
$$


where $p_{i}, 1 \leqq i \leqq 2$, are odd numbers greater than one, $h_{i}, 1 \leqq i \leqq 2$, are bounded and $\lambda, \mu \in R$ has at least one solution. (We must take $w_{1}(t)=\left(-t^{p_{1}}\right)^{p}, w_{2}(t)=\left(-t^{p 2}\right)^{p}$ and $p$ an odd number, $p>n$.)

Final remark. Our main result (Theorem 2.1) can always be applied to problems where condition $(\mathrm{H})$ is satisfied and not only to the problems considered in this section. For instance, if we have the B.V.P. (ordinary case)

$$
\begin{array}{cc}
u_{1}^{\prime \prime}-2 u_{1}-3 u_{2}=g_{1}\left(x, u_{1}, u_{2}\right), & x \in(0, \pi) \\
u_{2}^{\prime \prime}+2 u_{2}+u_{1}=g_{2}\left(x, u_{1}, u_{2}\right), & x \in(0, \pi) \\
u_{1}(0)=u_{1}(\pi)=u_{1}^{\prime}(0)+u_{1}^{\prime}(\pi)=0 ; & u_{2}(0)=u_{2}(\pi)=u_{2}^{\prime}(0)+u_{2}^{\prime}(\pi)=0
\end{array}
$$

then, it is not difficult to prove that $\phi_{1}(x)=\phi_{2}(x)=(\sin x) \sqrt{(2 / \pi)}$ and consequently (2.3) is satisfied.

Also we may consider systems of the form (3.1) with some boundary conditions of Dirichlet type and the others of Neumann type.

Acknowledgments. The author would like to express his thanks to the referee for his helpful comments and suggestions.

This work was supported in part by C.A.I.C.Y.T., Ministry of Education (Spain), under grant number 3258/83.

\section{REFERENCES}

1. R. A. Adams, Sobolev Spaces (Academic Press, 1975).

2. S. Agmon, A. Douglis and L. Nirengerg, Estimates near the boundary for solutions of elliptic partial differential equations satisfying general boundary conditions, I, Comm. Pure Appl. Math. 12 (1959), 623-727.

3. H. Brezis and W. A. Strauss, Semilinear second order elliptic equations in $L^{1}, J$. Math. Soc. Japan 25 (1973), 565-590.

4. A. Cañada and P. Martinez-Amores, Solvability of some operator equations and periodic solutions of nonlinear functional differential equations, J. Differential Equations 49(3) (1983), 415-429.

5. A. Cañada and R. Ortega, Existence theorems for equations in normed spaces and boundary value problems for nonlinear vector ordinary differential equations, Proc. Royal Soc. Edinburgh 98A (1984), 1-11.

6. A. CAÑada, $K$-set contractions and nonlinear vector boundary value problems, J. Math. Anal. Appl. 117 (1) (1986), 1-22.

7. D. G. DE Figueiredo and W. NI, Perturbations of second order linear elliptic problems by nonlinearities without Landesman-Lazer condition, Nonlinear Anal. 3(5) (1979), 295-307.

8. L. E. Fraenkel, On the embedding of $C^{1}(\Omega)$ in $C^{0+a}(\Omega)$, J. London Math. Soc. (2) 26 (1982), 290-298. 
9. S. Fucik and A. KufNer, Nonlinear Differential Equations (Elsevier, 1980).

10. L. J. Kazdan and F. W. Warner, Remarks on some quasilinear elliptic equations, Comm. Pure Appl. Math. 28 (1975), 567-597.

11. E. M. Landesman and A. C. Lazer, Nonlinear perturbations of linear elliptic boundary value problems at resonance, J. Math. Mech. 19 (1970), 609-623.

12. L. Mawhin, Problémes aux limites du type de Neumann pour certaines equations différentielles on aux dérivées partielles non linéaires, in Equations différentielles et fonctionnelles non linéaires (Herman, 1973), 124-134.

13. J. Mawhin, Topological degree methods in nonlinear boundary value problems, Amer. Math. Soc. Reg. Conf. in Math. 40 (1979).

14. N. Rouche and J. Mawhin, Equations differentielles ordinaires, Vol. II (Masson, 1973).

Departamento de Analisis Matematico

UnIVERSIDAD DE GRANADA

18071, Granada

SPAIN 